\title{
Detecting endogenous SUMO targets in mammalian cells and tissues
}

\author{
Janina Becker ${ }^{1}$, Sina V Barysch ${ }^{1}$, Samir Karaca $^{2}$, Claudia Dittner ${ }^{1,3}$, He-Hsuan Hsiao ${ }^{2}$, Mauricio Berriel Diaz $^{3}$, \\ Stephan Herzig ${ }^{3}$, Henning Urlaub ${ }^{2,4} \&$ Frauke Melchior $^{1}$
}

\begin{abstract}
SUMOylation is an essential modification that regulates hundreds of proteins in eukaryotic cells. Owing to its dynamic nature and low steady-state levels, endogenous SUMOylation is challenging to detect. Here, we present a method that allows efficient enrichment and identification of endogenous targets of SUMO1 and the nearly identical SUMO2 and 3 (SUMO 2/3) from vertebrate cells and complex organ tissue. Using monoclonal antibodies for which we mapped the epitope, we enriched SUMOylated proteins by immunoprecipitation and peptide elution. We used this approach in combination with MS to identify SUMOylated proteins, which resulted in the first direct comparison of the endogenous SUMO1- and SUMO2/3modified proteome in mammalian cells, to our knowledge. This protocol provides an affordable and feasible tool to investigate endogenous SUMOylation in primary cells, tissues and organs, and it will facilitate understanding of SUMO's role in physiology and disease.
\end{abstract}

SUMOylation is an essential post-translational modification that regulates protein functions. All eukaryotes express at least one small ubiquitin-related modifier (SUMO) protein; higher eukaryotes including plants and vertebrates express several SUMO paralogs belonging to two subfamilies (SUMO1 and SUMO2/3) that have overlapping and distinct targets and functions. Mammalian SUMO1 shares 50\% identity with SUMO2 and SUMO3, the latter of which are virtually identical. Hundreds of proteins are SUMOylated and deSUMOylated in a temporally and/or spatially controlled manner, such that selected targets may be SUMOylated only during a specific time in the cell cycle, in response to DNA damage or upon extracellular signals. Hence, SUMOylation contributes to numerous intracellular processes including transcription, DNA repair, chromatin remodeling and signal transduction (reviewed in refs. 1,2). Unsurprisingly, defects in SUMOylation have been associated with severe diseases such as cancer, neurodegeneration and heart failure (recent examples in refs. 3,4 ). Notably, numerous stress conditions are known to induce global changes in SUMOylation, both in tissue-cultured cells and at the organismic level. Examples for the latter are ischemia in the mouse brain ${ }^{5}$ or hibernation torpor in ground squirrels ${ }^{6,7}$. Studies in Arabidopsis thaliana suggest that these global changes are required for survival under adverse conditions ${ }^{8}$. Detailed understanding of SUMOylation, both at the level of individual target proteins and the systemic level is, hence, essential for understanding of physiological and pathophysiological processes. However, there are two major problems with the detection of SUMO targets. First, many SUMOylated proteins such as transcription factors are low in abundance. Because only a small fraction of these targets are normally SUMOylated at steady state, detection by direct immunoblotting is often impossible. Second, SUMOylated species are rapidly lost upon cell lysis in nondenaturing buffers, owing to highly active SUMO isopeptidases. Consequently, large efforts have been made during recent years to develop protocols and tools for the identification and analysis of rare SUMOylated proteins. It is a common strategy to overexpress tagged versions of SUMO along with a putative target protein in cells and evaluate target modification by immunoblotting or immunoprecipitation followed by immunoblotting ${ }^{9-12}$. To further boost SUMOylation, parts of the SUMOylation machinery (for example, the E2-conjugating enzyme Ubc9 or PIAS E3 ligases) can be coexpressed ${ }^{9,13-15}$. Although these strategies are useful to test whether proteins can in principle be SUMOylated and can help in development of SUMOylation-deficient protein variants, they obviously provide little insight into the endogenous regulation of target SUMOylation and are limited to transfectable material and genetically modifiable organisms.

Tagged SUMO has also been used successfully to identify new SUMO targets on a global scale. Expression of histidine (His)-tagged SUMO followed by Ni pulldown ${ }^{16-19}$ allows denaturing cell lysis, which inhibits SUMO isopeptidases and breaks up noncovalent protein interactions. Expression of hemagglutinin-tagged SUMO allows immunoprecipitation with peptide elution, which is known to have little background ${ }^{20-22}$. Combinations of Ni pulldown and immunoprecipitation have been performed in many model organisms, such as Saccharomyces cerevisiae ${ }^{23-26}$, Caenorhabditis elegans ${ }^{27}$, Drosophila melanogaster ${ }^{28}$ and $A$. thaliana ${ }^{29}$, which resulted in the identification of hundreds of putative SUMO targets in the respective model organisms. In mammalian cells, a tandem affinity purification (TAP) of

\footnotetext{
1Zentrum für Molekulare Biologie Heidelberg (ZMBH), DKFZ-ZMBH Alliance, Heidelberg, Germany. ${ }^{2}$ Max Planck Institute for Biophysical Chemistry, Göttingen, Germany. ${ }^{3}$ Joint Division Molecular Metabolic Control, Zentrum für Molekulare Biologie Heidelberg, Deutsches Krebsforschungszentrum (DKFZ) and University Hospital Heidelberg, DKFZ-ZMBH Alliance, Heidelberg, Germany. ${ }^{4}$ Department of Clinical Chemistry, University Medical Center Göttingen, Göttingen, Germany. Correspondence should be addressed to F.M. (f.melchior@zmbh.uni-heidelberg.de).
} 
TAP-SUMO2-modified proteins has been very successful ${ }^{30}$. Of note, several of the screens mentioned above were performed under stress conditions $^{21,22,24,29,30}$ that are known to enhance SUMOylation ${ }^{31}$.

Analysis of endogenous SUMOylation, however, either at the level of individual targets or at a global scale, has only been possible in isolated cases. A recently developed protocol that allows global enrichment of SUMOylated proteins from untransfected cells makes use of the poly-SUMO-binding function of RNF4, a protein containing four SUMO-interacting motifs (SIMs). Although it has been successfully used to identify proteins modified by SUMO2/3 from HeLa cells upon heat stress ${ }^{32}$, it is limited to proteins that are poly-SUMOylated.

Although numerous protocols are available, analysis of individual SUMOylated proteins in untransfected cell lines, primary cells, complex organs and tissues including human patient material remains rather challenging. We therefore developed a protocol that allows high enrichment of endogenously SUMOylated proteins from a wide range of vertebrate cells and tissues. It involves monoclonal antibodies to SUMO1 and SUMO2/3 that are readily available and is hence affordable and widely applicable both for the analysis of individual SUMOylated proteins and for global analysis of the SUMOylated proteome. As a proof of principle, we provide the first direct comparison, to our knowledge, of the SUMO1- and SUMO2/3-target proteomes in HeLa cells and demonstrate that endogenous SUMO targets can be efficiently enriched and identified from mouse liver. With this protocol, investigating the role of endogenous SUMOylation to answer physiological and disease-related questions will become feasible.

\section{RESULTS}

Enrichment of SUMOylated proteins with monoclonal antibodies To isolate endogenous SUMOylated species, we turned to two well-known monoclonal anti-SUMO1 and anti-SUMO2/3 antibodies (SUMO1 21C7 and SUMO2 8A2), whose hybridoma cells were developed previously 33,34 and can be obtained from the Developmental Studies Hybridoma Bank at the University of Iowa, Iowa City, Iowa, USA. These antibodies have similar ability to recognize the respective recombinant SUMO proteins in immunoblotting (Fig. 1a). We cultivated the hybridoma cells under conditions that allowed production of antibodies without contaminating bovine antibodies, immobilized the antibodies on protein G-agarose beads and optimized the conditions for immunoprecipitation of SUMOylated proteins. Asynchronously growing HeLa suspension cells were lysed in $1 \%$ SDS and $10 \mathrm{mM} N$-ethylmaleimide (NEM) to completely unfold and disrupt protein complexes and to inactivate SUMO isopeptidases. Sonication, heat denaturation in the presence of $50 \mathrm{mM}$ dithiothreitol to disrupt disulfide and thioester bonds and ten-fold dilution to establish radioimmunoprecipitation assay (RIPA) buffer conditions were required before the addition of immobilized antibodies (Online Methods). At this and all subsequent steps, fresh NEM was added. Beads were harvested and washed, and bound proteins were eluted with Laemmli buffer after overnight incubation with cell lysate. With this protocol (Fig. 1b), we were able to efficiently enrich endogenously SUMOylated proteins, as revealed by SDS-PAGE and subsequent immunoblotting with polyclonal antibodies to SUMO1 and SUMO2/3 (Fig. 1c). As expected for unstressed mammalian cells ${ }^{31}$, the most prominent band in the SUMO1 immunoprecipitation is SUMOylated RanGAP1 (migrating at $90 \mathrm{kDa}$ ), and the most prominent band in the SUMO2 immunoprecipitation is free SUMO2/3 (migrating at $20 \mathrm{kDa}$ ). Although these findings showed that the monoclonal antibodies SUMO1 21C7 and SUMO2 8A2 are well suited for our aim, staining with colloidal Coomassie blue revealed no differences in the total protein content of the SUMO and control immunoprecipitations (Fig. 1d). There was also no similarity between the overall protein pattern and the SUMO pattern (Fig. 1c compared to the S1 lane in Fig. 1d). This indicates high unspecific binding, which not only causes severe background problems in MS-based analyses but also limits how much sample can be loaded.

\section{Identification of epitope-spanning peptides}

A well-known method to reduce contaminations in immunoprecipitations is selective elution of antigens from their antibodies with epitope-containing peptides, and it is frequently used in protocols involving antibodies to hemagglutinin or Flag tags ${ }^{35-37}$. As the epitopes recognized by the monoclonal antibodies SUMO1 $21 \mathrm{C} 7$ and SUMO2 8A were unknown, we generated overlapping peptides covering the entire SUMO sequences and performed peptide-competition assays (Fig. 2a,b). Once competing peptides were identified, we tested shorter variants to identify the minimal epitope-spanning peptides (data not shown). The peptides SUMO1 57-VPMNSLRFLFE-67 and SUMO2 57-IRFRFDGQPI-66 contain the epitope for the monoclonal antibodies SUMO1 $21 \mathrm{C} 7$ and SUMO2 8A2, respectively.

Comparison of the epitope-spanning sequences in SUMO proteins from different species showed that the antibodies recognize SUMO in a range of model organisms. For SUMO1 21C7, the epitope is identical in humans, mice, chickens and Xenopus laevis (Fig. 2a). Although zebrafish SUMO1 does not share full homology in this part of the sequence, additional peptide competition assays indicate that it is also recognized by the antibody (Supplementary Fig. 1), whereas C. elegans SUMO1 is too divergent. For SUMO2 8A2, the epitope is identical in humans, mice, chickens, X. laevis and zebrafish (Fig. 2b); D. melanogaster SUMO2, whose epitope-spanning region differs in one amino acid, is also recognized by the antibody, both in competition assays and in immunoblotting (Supplementary Fig. 1).
Figure 1 Two well-known monoclonal antibodies work efficiently in denaturing SUMO1 and SUMO2/3 immunoprecipitation. (a) Immunoblots comparing the sensitivity of SUMO1 $21 \mathrm{C} 7$ and SUMO2 8A2 mouse monoclonal antibodies toward recombinant SUMO1 and SUMO2. (b) Flow chart for the immunoprecipitation. (c) Immunoblots showing immunoprecipitations (IP) performed with immobilized SUMO1 21C7, SUMO2 8A2 or normal mouse IgG as control. Immunoblot analyses were performed with rabbit antiSUMO1 and rabbit anti-SUMO2 antibodies. (d) Colloidal Coomassie staining of SUMO control immunoprecipitations, revealing high bac control; I, input; M, marker, MW, molecular weight.



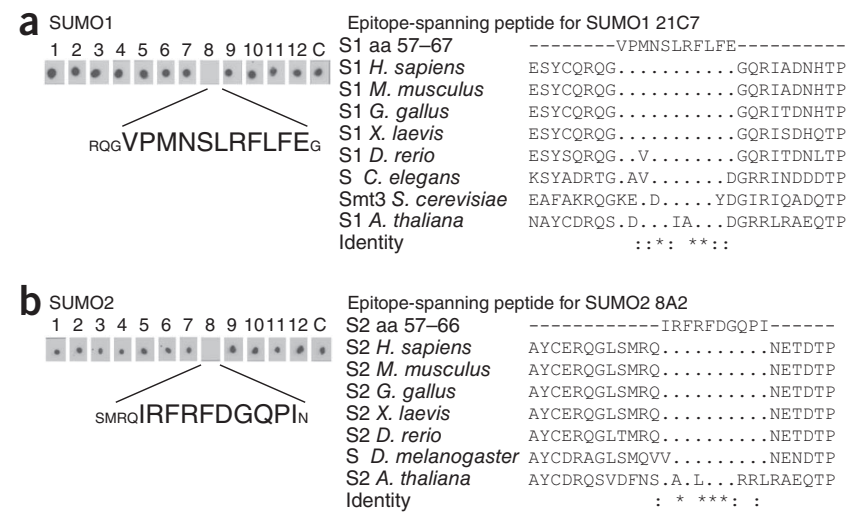

Establishing an efficient peptide elution protocol

Having defined minimal epitope-spanning peptides, we next established efficient peptide elution conditions. A systematic analysis, including variations in $\mathrm{pH}$, temperature, salt and detergent types, resulted in an elution protocol using RIPA buffer with $500 \mathrm{mM}$ salt (Online Methods) for $30 \mathrm{~min}$ at $37^{\circ} \mathrm{C}$. With these conditions established, we repeated the immunoprecipitation from denatured HeLa lysates but used peptide elution rather than elution with Laemmli buffer (Fig. 3). To test the specificity of the peptide elution, we employed three conditions for each immunoprecipitation: (i) antiSUMO-antibody beads for elution with the corresponding epitopespanning peptides, (ii) anti-SUMO-antibody beads for elution with a control peptide and (iii) control beads for elution with the epitopespanning peptides. To evaluate the efficiency and specificity of the protocol, we analyzed samples by immunoblotting with polyclonal anti-SUMO antibodies. Both immunoprecipitation and peptide elution are efficient and specifically enrich SUMOylated proteins (Fig. 3a,b). Of note, the small fraction of free SUMO2/3 that is visible in the eluate with control peptide is a consequence of the stringent elution conditions (buffer and elevated temperature) needed for efficient elution from the beads.

To test whether inclusion of the peptideelution step solves the problem of nonspecific

Figure 3 Enrichment of endogenously SUMOylated proteins by immunoprecipitation and peptide elution from HeLa cells. Immunoprecipitations involve the monoclonal antibodies SUMO1 $21 \mathrm{C} 7$ and SUMO2 8A2 or normal mouse IgG as control. Elution was done with the epitope-containing peptides (epi) or with control peptides (con). (a,b) Immunoblots with rabbit anti-SUMO1 and rabbit antiSUMO2 antibodies, revealing efficiency of the immunoprecipitations and the peptide elutions. (c) Colloidal Coomassie staining of SUMO and control immunoprecipitations, revealing low background contamination after peptide elution. (d) Colloidal Coomassie staining of SUMO2 immunoprecipitations from control cells and cells heat shocked for $30 \mathrm{~min}$ at $42{ }^{\circ} \mathrm{C}(\mathrm{HS})$, revealing the expected decrease of free species and increase of higher-molecularweight species. Throughout figure, I, input; FT, flow through; E, eluate; B, beads after elution; S1, SUMO1; S2, SUMO2; C, control; asterisk, cross-reacting band; M, marker. a

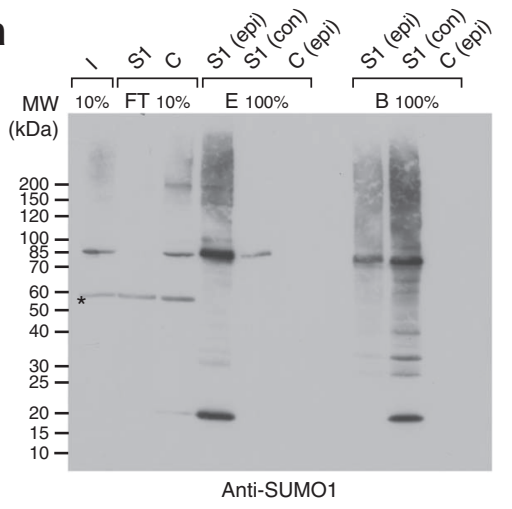

C

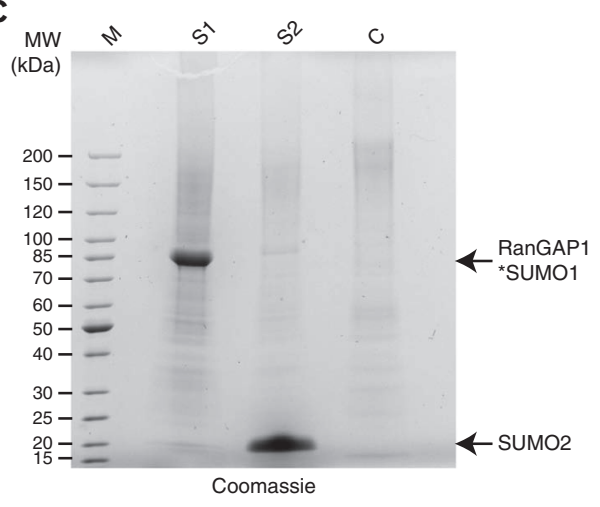

Figure 2 Identification of epitope-spanning peptides for monoclonal SUMO1 21C7 and SUMO2 8A2 antibodies. (a,b) Left, dot blots of immobilized SUMO probed with SUMO peptide-preincubated antiSUMO antibodies SUMO1 21C7 (a) and SUMO2 8A2 (b). Overlapping peptides (15-mers) covering the entire SUMO1 and SUMO2 amino acid sequence were tested for their ability to compete with immobilized SUMO in immunoblotting. Large letters indicate the minimal epitopespanning peptides, as determined by secondary screens (not shown). Right, alignment of minimal peptide sequences with SUMO sequences of different species. aa, amino acid. Asterisk denotes identical residues; colon denotes residues with similar properties.

contaminants in the SUMO immunoprecipitations, we performed large-scale experiments with HeLa cells, followed by peptide elution, trichloroacetic acid precipitation, SDS-PAGE and colloidal Coomassie staining (Fig. 3c). Indeed, in contrast to the SDS elution (Fig. 1d), the colloidal Coomassie staining after peptide elution resembles the signals in SUMO immunoblotting. In the SUMO1 immunoprecipitation, SUMO1-RanGAP1 was clearly visible as the most prominent band, and a high-molecular-weight smear could be detected in the whole lane. As revealed by densitometry, the intensity of the high-molecular-weight smear was nearly twice that of the control eluate, which indicated that a substantial proportion of the precipitated proteins was specific for the SUMO1 immunoprecipitation. In the SUMO2 immunoprecipitation, free SUMO2 was the strongest band, and a higher-molecular-weight smear of the conjugates was visible as well. To obtain further evidence that the Coomassie signal observed upon SUMO2 immunoprecipitations at least reflects most, if not all, SUMOylated proteins, we also compared SUMO2 immunoprecipitations from unstressed HeLa suspension cells to those subjected to heat shock (Fig. 3d). As expected, free SUMO2/3 decreased, and the signal intensity in the highermolecular-weight range increased. In conclusion, the amount of unspecific proteins could be substantially reduced in the SUMO immunoprecipitation protocol that includes peptide elution. 
a



Figure 4 A new monoclonal anti-SUMO1 antibody works efficiently in immunoprecipitation and peptide elution. (a) Alignment of the minimal epitope-spanning peptide for SUMO1 $1_{76-86}$ with SUMO sequences of different species. Asterisk denotes identical residues; colon denotes residues with similar properties. (b) Comparison of SUMO1 immunoprecipitations and peptide elutions performed with SUMO1 21C7 and SUMO1 $1_{76-86}$. I, input; FT, flow through; E, eluate; S1, SUMO1; S2, SUMO2; C, control; asterisk, cross-reacting band.

Applying the protocol to a new monoclonal anti-SUMO1 antibody One remaining limitation of our protocol is that the monoclonal antibodies do not work with all species and may recognize some proteins that are not SUMOylated, owing to shared epitopes. To expand the list of useful anti-SUMO antibodies, we developed a new monoclonal antibody that was raised against a C-terminal peptide of SUMO1 (SUMO1 ${ }_{76-86}$; Online Methods). We mapped the minimal epitope-spanning peptide to SUMO1 76-TPKELGMEEED-86, which is identical in humans, mice, chickens and X. laevis (Fig. 4a). To test whether our immunoprecipitation and peptide elution protocol permits exchange of the antibody without further need for optimization, we repeated the experiment shown in Figure 3a with SUMO1 $1_{76-86}$. Indeed, the protocol works efficiently, as judged by the strong depletion of SUMO1 species in the flow through and the large recovery after peptide elution (Fig. $\mathbf{4 b}$ ).

Figure 5 Analysis of the endogenous SUMO1and SUMO2/3-modified proteome of HeLa cells. SUMOylated proteins enriched from asynchronously growing HeLa suspension cells by immunoprecipitations and peptide elutions were identified by MS analysis.

(a) Diagram comparing the relative abundances of 232 different SUMO candidates in control, SUMO1 and SUMO2/3 immunoprecipitations. Abundance was calculated on the basis of peptide signal intensities; complete data are in Supplementary Table 1 (complete list, 0-10\%). (b) Immunoblots showing the SUMO signals of large-scale SUMO immunoprecipitations. (c) Immunoblots of large-scale SUMO immunoprecipitations probed with the indicated antibodies to targets identified by MS analysis. Throughout figure, I, input; FT, flow through; E, eluate; S1, SUMO1; S2, SUMO2; C, control.
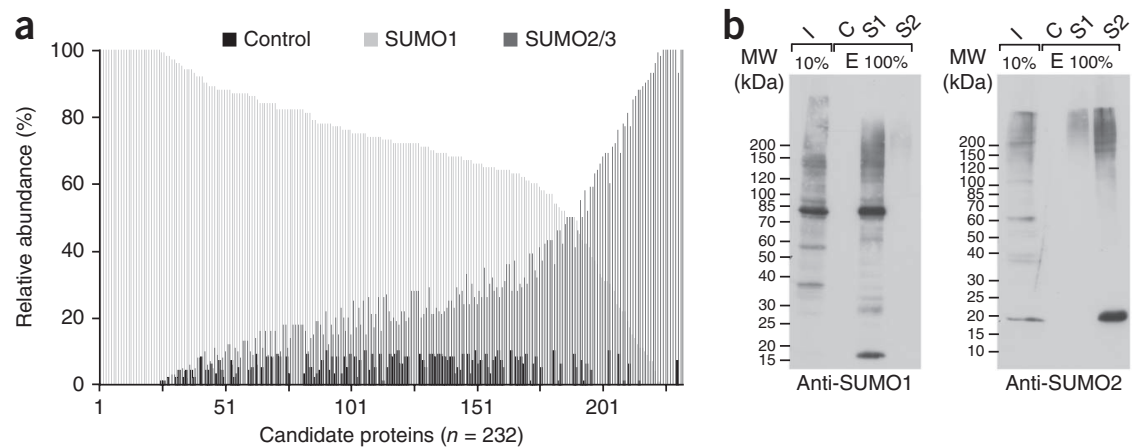

C


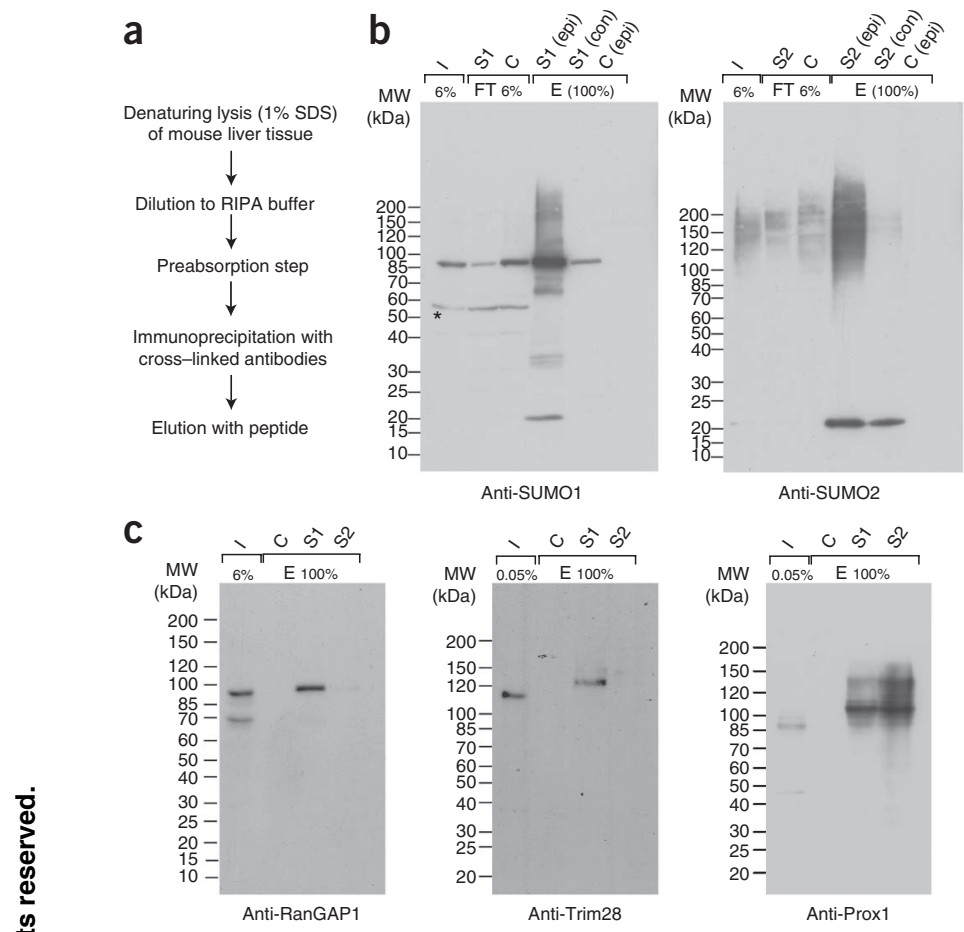

Figure 6 Endogenous SUMO targets can be enriched efficiently from mouse liver. (a) Flow chart of the experimental procedure. Immunoprecipitations involved monoclonal antibodies SUMO1 21C7 and SUMO2 8A2 or normal mouse IgG as control. Elution was done with epitope-containing peptides (epi) or with control peptides (con). (b) Immunoblots with rabbit anti-SUMO1 and rabbit anti-SUMO2 antibodies to control for efficiency of the immunoprecipitation and elution protocol. (c) Immunoblots of SUMO immunoprecipitations showing endogenous RanGAP1, Trim28 and Prox1 SUMOylated in mouse liver. Throughout figure, I, input; FT, flow through; E, eluate; S1, SUMO1; S2, SUMO2; C, control; asterisk, cross-reacting band.

anti-SUMO1 and the anti-SUMO2/3 antibodies compared to the control, $40 \%$ of all candidates showed enrichment in the SUMO1 immunoprecipitation, and $10 \%$ were found predominantly in the SUMO2/3 immunoprecipitation ( $>80 \%$ of total SUMO signal). Signal-intensity values for a selection of proteins are depicted in Table 1. For example, the well-known SUMO1 target RanGAP1 was found predominantly in the SUMO1 immunoprecipitation ${ }^{33,38}$. The SUMO targets $\mathrm{PML}^{39}$, Trim28 (ref. 40; also known as TIF1 $\beta$ and Kap1), TFII-I ${ }^{20}$ and Ubc9 (ref. 41), in contrast, showed a clear enrichment in the immunoprecipitation of SUMO2/3.

To verify this paralog selectivity as determined by MS, we analyzed samples from large-scale immunoprecipitations (Fig. 5b) in immunoblotting experiments. Both SUMOylation as well as paralog selectivity of all known SUMO targets could be confirmed (Fig. 5c). We also verified the SUMOylation of two newly identified targets, the splicing factor RBM25 (ref. 42) and the zinc-finger protein WIZ ${ }^{43}$ (Fig. 5c). Whereas WIZ also showed the expected paralog preference, the signals obtained for RBM25 in immunoblotting (reproducible preference for SUMO1; faint signal for SUMO2 in long exposures) deviated from those obtained by MS (similar intensities for SUMO1 and SUMO2/3). A possible explanation of this apparent discrepancy is the difference between both methods: whereas values obtained from the MS analysis reflect the sum of peptides in the whole gel, in immunoblotting, faint high-molecular-weight bands that would derive from SUMO2/3 chains may be below the detection level.
Table 1 Relative distribution of selected SUMO candidates in control, SUMO1 21C7 and SUMO2 8A2 immunoprecipitates

\begin{tabular}{|c|c|c|c|}
\hline Protein names & Control (\%) & SUMO1 (\%) & SUMO2 (\%) \\
\hline $\begin{array}{l}\text { Ran GTPase-activating protein } 1 \\
\text { (RanGAP1) }\end{array}$ & 0 & 84 & 16 \\
\hline Tripartite motif protein TRIM19 $\kappa(P M L)$ & 0 & 7 & 93 \\
\hline $\begin{array}{l}\text { Transcription intermediary factor } \\
1-\beta \text { (Trim28) }\end{array}$ & 0 & 33 & 66 \\
\hline General transcription factor II-I (TFII-I) & 0 & 28 & 72 \\
\hline SUMO-conjugating enzyme UBC9 & 0 & 0 & 100 \\
\hline RNA-binding protein 25 (RBM25) & 1 & 42 & 58 \\
\hline WIZ & 0 & 73 & 27 \\
\hline
\end{tabular}

Percentages are derived from protein intensity values calculated from summed signal intensities of the identified peptides by MaxQuant.

From that, we conclude that our immunoprecipitation and peptide elution protocol is a reliable tool to enrich and verify endogenously SUMOylated proteins, including those that were previously very difficult to detect, such as SUMOylated Ubc9 (ref. 41).

\section{Enrichment of SUMOylated proteins from liver tissue}

To cover a broad range of applications, we tested whether our protocol can enrich SUMOylated proteins from a complex tissue like mouse liver. Although most steps could be applied from the immunoprecipitation protocol for HeLa cells, an additional step for the preabsorption of antibodies (which are intrinsically present in the lysates of blood vessel-containing organs) had to be included (Fig. 6a). Successful SUMO1 and SUMO2 immunoprecipitations were obtained from mouse liver extracts (Fig. 6b). Similar to the results obtained for HeLa cells (Fig. 3a,b), depletion was observed in the flow-through sample after immunoprecipitation with anti-SUMO but not control antibodies and strong enrichment of the SUMO signal in the eluate with the epitope-spanning (epi) but not the control (con) peptide. We next tested whether our protocol is applicable to verification of specific SUMO targets in mouse liver and were able to confirm endogenous SUMOylation of the known targets RanGAP1 (refs. 33,38), Trim 28 (ref. 40) and Prox1 (ref. 44) by immunoblotting (Fig. 6c). These experiments clearly demonstrate that our protocol is universally applicable to study endogenous SUMOylation.

\section{DISCUSSION}

Although SUMOylation is one of the major regulatory protein modifications, tools for the analysis of endogenous SUMOylation are still scarce. Here, we provide a method that allows efficient enrichment of endogenously mono- or poly-SUMOylated proteins, both from cell lines and complex organ tissue. With the currently available antibodies, the protocol should be applicable to all vertebrates (including mammals, fish, birds and amphibians) and also to Drosophila (Fig. 2 and Supplementary Fig. 1), and it can probably be expanded to any other biological material as soon as the respective monoclonal SUMO antibodies and corresponding epitope-spanning peptides become available.

\section{Analysis of the SUMO proteome}

Here, we provide what is, to our knowledge, the first endogenous SUMO1- and SUMO2/3-modified proteome candidate lists from asynchronously growing HeLa suspension cells, which includes 584 different proteins (Supplementary Table 1). How do these putative targets compare to verified SUMO targets and candidates from other proteomic approaches? First, at least 30 proteins (13\%) on our highstringency list (232 proteins with less than $10 \%$ signal in the control 
immunoprecipitation) have previously been described as SUMO targets in mammalian cells (references in Supplementary Table 1). Second, direct comparison with a recent study performed in mammalian cells without stress stimuli ${ }^{18}$ indicates a clear overlap: upon expression of a modified version of His-SUMO2, a previous study was able to identify SUMOylation sites in 80 different targets, of which we identified 27 in our screen. Third, a recent study identified 76 candidates of poly-SUMOylation in unstressed HeLa cells and 972 candidates upon heat shock ${ }^{32}$. As shown in Supplementary Figure 2, little overlap exists between the 'no-heat-shock' candidates and our lists (6 of our 232 (3\%) or 33 of our 584 (6\%) candidates). However, a large overlap exists between our lists and the 'heat-shock' list: 92 of 232 (40\%) or 296 of 584 (51\%) candidates that we identified in the absence of stress were identified in ref. 32 upon heat shock (Supplementary Table 2). This indicates that many proteins subject to chain formation of SUMO2/3 upon heat shock are already monoSUMOylated in the absence of stress. Taken together, our own validation (Fig. 5) and the comparison with different studies confirm the credibility of our list of 584 endogenous SUMO target candidates and the overall quality of the method.

\section{Paralog-specific SUMOylation}

As described above, our data indicate that most SUMO targets in asynchronously growing HeLa suspension cells are modified with both SUMO1 and SUMO2/3, however with highly variable relative ratios (Fig. 5a). Of all SUMO targets, $40 \%$ seem preferentially modified with SUMO1 and only $10 \%$ with SUMO2/3. In light of a previous study with stable HeLa cell lines expressing His-SUMO ${ }^{17}$, our finding may appear surprising. The study found 55 SUMO targets, with 25 being preferentially modified with SUMO1, 19 with SUMO2 and only $9(16 \%)$ similarly modified with both paralogs. A key difference from our study, however, is that the previous study compared two different stable cell lines overexpressing His-tagged SUMO1 and SUMO2. Because endogenous SUMO was still present, both the relative levels of SUMO1 versus SUMO2/3 and the ratio between endogenous and tagged SUMO paralogs was different. Although our protocol requires two different monoclonal antibodies (which could possibly give rise to different contaminants that would be identified as paralog specific), it has the strong advantage that a single cell extract can be used for both immunoprecipitations. This allows for direct comparison by MS, immunoprecipitation and immunoblotting, as long as SUMO1 and SUMO2/3 targets are enriched with comparable efficiency.

Assuming that many proteins have the potential to be SUMOylated by both SUMO variants, factors that contribute to paralog-specific modification are the relative abundance of SUMO1 and SUMO2/3 proteins and relative isopeptidase activities. In line with this idea, SUMO2/3 can compensate for loss of SUMO1 in a knockout mouse model ${ }^{45}$. Of particular interest, however, are those proteins that show a striking preference for SUMO1 or SUMO2/3. Three mechanisms concerning their paralog preference have been described. It might originate from an E3 ligase, as is the case for the small E3 ligase fragment of RanBP2 that carries a SIM to facilitate recruitment of SUMO1loaded Ubc9 (refs. 46,47). Similarly, the preference may be caused by a SIM in the target (SIM-dependent SUMOylation), as has been suggested for the DNA helicase BLM ${ }^{48}$ and the ubiquitin-specific protease Usp25 (ref. 49), both of which are preferentially modified with SUMO2/3. Finally, a protein may be modified with SUMO1 and with SUMO2/3, but selective protection of one SUMOylated species from SUMO isopeptidases may allow this form to accumulate. Such a mechanism explains the marked preference of RanGAP1 for SUMO1, which can form an isopeptidase-resistant complex with RanBP2 and
Ubc9 only when modified with SUMO1 but not when modified with SUMO2/3 (refs. 50,51). The list presented here of proteins that show a preference for SUMO1 or SUMO2/3 offer an ideal resource for investigating additional mechanisms underlying paralog specificity.

\section{Outlook}

We described a universally applicable and affordable method to identify and investigate individual endogenously SUMOylated proteins as well as the endogenous SUMO proteome. It could be easily combined with second purification steps, for example to study proteins carrying two modifications simultaneously, and is suitable for quantitative approaches such as MS after stable isotope labeling by amino acids in cell culture (SILAC) labeling. Our method will thus greatly facilitate the analysis of one of the most common post-translational modifications.

\section{METHODS}

Methods and any associated references are available in the online version of the paper.

Note: Supplementary information is available in the online version of the paper.

\section{ACKNOWLEDGMENTS}

We gratefully acknowledge M. Matunis (Department of Biochemistry and Molecular Biology, Bloomberg School of Public Health, Johns Hopkins University, Baltimore, Maryland, USA) for making hybridomas for monoclonal anti-SUMO antibodies available to the community and H. de Thé (Université Paris Diderot, Sorbonne Paris Cité, Hôpital St. Louis 1, Paris, France) for generously providing anti-PML antibodies, R. Ramaker and N. Stankovic for help with antibody characterization and all lab members for sharing reagents and advice. This work was supported by the Deutsche Forschungsgemeinschaft (SPP1365, ME 2279/3 to F.M.) and the EU Network of Excellence Rubicon (to F.M.) and by fellowships of the Excellence Cluster CellNetworks (to S.V.B.) and the Deutsche Krebshilfe (to S.H.).

\section{AUTHOR CONTRIBUTIONS}

J.B. and S.V.B. designed and carried out most experiments and wrote the manuscript. C.D. carried out experiments and wrote the manuscript. S.K., H.-H.H. and H.U. carried out MS and data analyses. M.B.D. and S.H. helped with mouse liver experiments. F.M. guided the project, designed experiments and wrote the manuscript.

\section{COMPETING FINANCIAL INTERESTS}

The authors declare no competing financial interests.

Reprints and permissions information is available online at http://www.nature.com/ reprints/index.html.

1. Geiss-Friedlander, R. \& Melchior, F. Concepts in sumoylation: a decade on Nat. Rev. Mol. Cell Biol. 8, 947-956 (2007).

2. Gareau, J.R. \& Lima, C.D. The SUMO pathway: emerging mechanisms that shape specificity, conjugation and recognition. Nat. Rev. Mol. Cell Biol. 11, 861-871 (2010).

3. Kho, C. et al. SUMO1-dependent modulation of SERCA2a in heart failure. Nature 477, 601-605 (2011).

4. Bertolotto, C. et al. A SUMOylation-defective MITF germline mutation predisposes to melanoma and renal carcinoma. Nature 480, 94-98 (2011).

5. Datwyler, A.L. et al. SUMO2/3 conjugation is an endogenous neuroprotective mechanism. J. Cereb. Blood Flow Metab. 31, 2152-2159 (2011).

6. Lee, Y.J. \& Hallenbeck, J.M. Insights into cytoprotection from ground squirrel hibernation, a natural model of tolerance to profound brain oligaemia. Biochem. Soc. Trans. 34, 1295-1298 (2006).

7. Lee, Y.J. et al. Protein SUMOylation is massively increased in hibernation torpor and is critical for the cytoprotection provided by ischemic preconditioning and hypothermia in SHSY5Y cells. J. Cereb. Blood Flow Metab. 27, 950-962 (2007).

8. Conti, L., Kioumourtzoglou, D., O'Donnell, E., Dominy, P. \& Sadanandom, A. OTS1 and OTS2 SUMO proteases link plant development and survival under salt stress. Plant Signal. Behav. 4, 225-227 (2009).

9. Desterro, J.M., Rodriguez, M.S. \& Hay, R.T. SUMO-1 modification of $\mathrm{I} \kappa \mathrm{B} \alpha$ inhibits NF-KB activation. Mol. Cell 2, 233-239 (1998). 
10. Hong, Y. et al. SUMOylation of DEC1 protein regulates its transcriptional activity and enhances its stability. PLOS ONE 6, e23046 (2011).

11. Muller, S. et al. c-Jun and p53 activity is modulated by SUMO-1 modification. J. Biol. Chem. 275, 13321-13329 (2000).

12. Rodriguez, M.S. et al. SUMO-1 modification activates the transcriptional response of p53. EMBO J. 18, 6455-6461 (1999).

13. Kjenseth, A. et al. The gap junction channel protein connexin43 is covalently modified and regulated by SUMOylation. J. Biol. Chem. 287, 15851-15861 (2012).

14. Ismail, I.H. et al. CBX4-mediated SUMO modification regulates BMI1 recruitment at sites of DNA damage. Nucleic Acids Res. 40, 5497-5510 (2012).

15. Yu, J. et al. RhoGDI SUMOylation at K138 increases its binding activity to Rho GTPase and its inhibiting cancer cell motility. J. Biol. Chem. 287, 13752-13760 (2012).

16. Vertegaal, A.C. et al. A proteomic study of SUMO-2 target proteins. J. Biol. Chem. 279, 33791-33798 (2004).

17. Vertegaal, A.C. et al. Distinct and overlapping sets of SUMO-1 and SUMO-2 target proteins revealed by quantitative proteomics. Mol. Cell Proteomics 5, 2298-2310 (2006).

18. Matic, I. et al. Site-specific identification of SUMO-2 targets in cells reveals an inverted SUMOylation motif and a hydrophobic cluster SUMOylation motif. Mol. Cell 39, 641-652 (2010).

19. Galisson, F. et al. A novel proteomics approach to identify SUMOylated proteins and their modification sites in human cells. Mol. Cell Proteomics 10, M110.004796 (2011).

20. Zhao, Y., Kwon, S.W., Anselmo, A., Kaur, K. \& White, M.A. Broad spectrum identification of cellular small ubiquitin-related modifier (SUMO) substrate proteins. J. Biol. Chem. 279, 20999-21002 (2004).

21. Blomster, H.A. et al. Novel proteomics strategy brings insight into the prevalence of SUMO-2 target sites. Mol. Cell Proteomics 8, 1382-1390 (2009).

22. Yang, W. et al. Analysis of oxygen/glucose-deprivation-induced changes in SUMO3 conjugation using SILAC-based quantitative proteomics. J. Proteome Res. 11, 1108-1117 (2012).

23. Hoege, C., Pfander, B., Moldovan, G.L., Pyrowolakis, G. \& Jentsch, S. RAD6dependent DNA repair is linked to modification of PCNA by ubiquitin and SUMO. Nature 419, 135-141 (2002).

24. Zhou, W., Ryan, J.J. \& Zhou, H. Global analyses of sumoylated proteins in Saccharomyces cerevisiae. Induction of protein sumoylation by cellular stresses. J. Biol. Chem. 279, 32262-32268 (2004).

25. Denison, C. et al. A proteomic strategy for gaining insights into protein sumoylation in yeast. Mol. Cell Proteomics 4, 246-254 (2005).

26. Panse, V.G., Hardeland, U., Werner, T., Kuster, B. \& Hurt, E. A proteome-wide approach identifies sumoylated substrate proteins in yeast. J. Biol. Chem. 279, 41346-41351 (2004)

27. Kaminsky, R. et al. SUMO regulates the assembly and function of a cytoplasmic intermediate filament protein in C. elegans. Dev. Cell 17, 724-735 (2009).

28. Nie, M., Xie, Y., Loo, J.A. \& Courey, A.J. Genetic and proteomic evidence for roles of Drosophila SUMO in cell cycle control, Ras signaling, and early pattern formation. PLOS ONE 4, e5905 (2009).

29. Miller, M.J., Barrett-Wilt, G.A., Hua, Z. \& Vierstra, R.D. Proteomic analyses identify a diverse array of nuclear processes affected by small ubiquitin-like modifier conjugation in Arabidopsis. Proc. Natl. Acad. Sci. USA 107, 16512-16517 (2010).

30. Golebiowski, F. et al. System-wide changes to SUMO modifications in response to heat shock. Sci. Signal. 2, ra24 (2009).

31. Saitoh, H. \& Hinchey, J. Functional heterogeneity of small ubiquitin-related protein modifiers SUMO-1 versus SUMO-2/3. J. Biol. Chem. 275, 6252-6258 (2000).
32. Bruderer, R. et al. Purification and identification of endogenous polySUMO conjugates. EMBO Rep. 12, 142-148 (2011).

33. Matunis, M.J., Coutavas, E. \& Blobel, G. A novel ubiquitin-like modification modulates the partitioning of the Ran-GTPase-activating protein RanGAP1 between the cytosol and the nuclear pore complex. J. Cell Biol. 135, 1457-1470 (1996).

34. Zhang, X.D. et al. SUMO-2/3 modification and binding regulate the association of CENP-E with kinetochores and progression through mitosis. Mol. Cell 29, 729-741 (2008).

35. Einhauer, A. \& Jungbauer, A. The FLAG peptide, a versatile fusion tag for the purification of recombinant proteins. J. Biochem. Biophys. Methods 49, 455-465 (2001).

36. Field, J. et al. Purification of a RAS-responsive adenylyl cyclase complex from Saccharomyces cerevisiae by use of an epitope addition method. Mol. Cell Biol. 8 , 2159-2165 (1988)

37. Hopp, T.P. et al. A short polypeptide marker sequence useful for recombinant protein identification and purification. Nat. Biotechnol. 6, 1204-1210 (1988).

38. Mahajan, R., Delphin, C., Guan, T., Gerace, L. \& Melchior, F. A small ubiquitinrelated polypeptide involved in targeting RanGAP1 to nuclear pore complex protein RanBP2. Cell 88, 97-107 (1997).

39. Sternsdorf, T., Jensen, K. \& Will, H. Evidence for covalent modification of the nuclear dot-associated proteins PML and Sp100 by PIC1/SUMO-1. J. Cell Biol. 139, 1621-1634 (1997).

40. Lee, Y.K., Thomas, S.N., Yang, A.J. \& Ann, D.K. Doxorubicin down-regulates Kruppel-associated box domain-associated protein 1 sumoylation that relieves its transcription repression on p21WAF1/CIP1 in breast cancer MCF-7 cells. J. Biol. Chem. 282, 1595-1606 (2007)

41. Knipscheer, P. et al. Ubc9 sumoylation regulates SUMO target discrimination. Mol. Cell 31, 371-382 (2008).

42. Zhou, A., Ou, A.C., Cho, A., Benz, E.J. Jr. \& Huang, S.C. Novel splicing factor RBM25 modulates Bcl-x pre-mRNA 5' splice site selection. Mol. Cell Biol. 28, 5924-5936 (2008)

43. Ueda, J., Tachibana, M., Ikura, T. \& Shinkai, Y. Zinc finger protein Wiz links G9a/ GLP histone methyltransferases to the co-repressor molecule CtBP. J. Biol. Chem. 281, 20120-20128 (2006).

44. Shan, S.F. et al. Modulation of transcriptional corepressor activity of prospero-related homeobox protein (Prox1) by SUMO modification. FEBS Lett. 582, 3723-3728 (2008).

45. Evdokimov, E., Sharma, P., Lockett, S.J., Lualdi, M. \& Kuehn, M.R. Loss of SUMO1 in mice affects RanGAP1 localization and formation of PML nuclear bodies, but is not lethal as it can be compensated by SUMO2 or SUMO3. J. Cell Sci. 121 , 4106-4113 (2008).

46. Reverter, D. \& Lima, C.D. Insights into E3 ligase activity revealed by a SUMORanGAP1-Ubc9-Nup358 complex. Nature 435, 687-692 (2005).

47. Tatham, M.H. et al. Unique binding interactions among Ubc9, SUMO and RanBP2 reveal a mechanism for SUMO paralog selection. Nat. Struct. Mol. Biol. 12, 67-74 (2005).

48. Zhu, J. et al. Small ubiquitin-related modifier (SUMO) binding determines substrate recognition and paralog-selective SUMO modification. J. Biol. Chem. 283, 2940529415 (2008).

49. Meulmeester, E., Kunze, M., Hsiao, H.H., Urlaub, H. \& Melchior, F. Mechanism and consequences for paralog-specific sumoylation of ubiquitin-specific protease 25. Mol. Cell 30, 610-619 (2008).

50. Zhu, S. et al. Protection from isopeptidase-mediated deconjugation regulates paralog-selective sumoylation of RanGAP1. Mol. Cell 33, 570-580 (2009).

51. Werner, A., Flotho, A. \& Melchior, F. The RanBP2/RanGAP1(*)SUMO1/Ubc9 complex is a multisubunit SUMO E3 ligase. Mol. Cell 46, 287-298 (2012). 


\section{ONLINE METHODS}

Peptides. Peptides for epitope mapping and peptide elution were synthesized by the peptide-synthesis unit of the German Cancer Research Center. Stock solutions of $10 \mathrm{mg} / \mathrm{ml}$ in DMSO were stored at $-80^{\circ} \mathrm{C}$.

Antibodies. Mouse hybridoma cells producing anti-SUMO1 and anti-SUMO2/3 antibodies were ordered from the Developmental Studies Hybridoma Bank at the University of Iowa (SUMO1 21C7, SUMO2 8A2). Hybridoma cells for monoclonal anti-SUMO $\mathrm{SU}_{176-86}$ antibody were generated upon immunizing mice with the carrier-coupled peptide CIADNHTPKLELGMEEEDVIEVYQEQT by using a commercial service (BioGenes $\mathrm{GmBH}$ ). The minimal epitope-spanning peptide was mapped by competition experiments (described below) with the peptide TPKELGMEEED. Rabbit anti-SUMO1 (1:1,000) and goat anti-RanGAP1 antibodies $(1: 1,000)$ have been described previously ${ }^{38,52}$. Chicken anti-PML antibody $(1: 1,000)$ was from $\mathrm{H}$. de Thé5 ${ }^{33}$. Commercial antibodies used were rabbit anti-SUMO2 (Eurogentec, cat. no. AV-SM23-0100, 1:1000), rabbit anti-Trim28 (Cell Signaling, cat. no. 4124, 1:1,000) and rabbit anti-TFII-I (Cell Signaling, cat. no. 4562, 1:1,000), rabbit anti-RBM25 (Novus Biologicals, cat. no. NB100-57505, 1:2,000), goat anti-WIZ (Acris Antibodies, cat. no. AP16897PU-N, 1:500), rabbit anti-Prox1 (Upstate, cat. no. 07-537, 1:2,000), rabbit anti-Ubc9 (Santa Cruz, cat. no. SC-10759, 1:1,000) and secondary peroxidase-conjugated donkey antibodies (Jackson Immuno Research).

Monoclonal anti-SUMO antibody production. A CELLine bioreactor (Integra Biosciences) was inoculated with the hybridoma cells, and the monoclonal antibodies were harvested from the bioreactor following the manufacturer guidelines, whereby the inner (cell and antibody) compartment was supplemented with hybridoma-SFM medium and the outer (medium) compartment with RPMI 1640 medium supplemented with 10\% heat-inactivated FBS and penicillin-streptomycin.

Immobilization of antibodies. Antibody ( $8 \mathrm{mg}$ of monoclonal anti-SUMO1, monoclonal anti-SUMO2 or normal mouse IgG (Invitrogen)) was added to $1 \mathrm{ml}$ of Protein G-agarose (Roche) equilibrated in $20 \mathrm{mM} \mathrm{NaP}$, pH 7.0. To cross-link the antibodies to Protein G, $50 \mathrm{mM}$ borate buffer, pH 9.0, containing $20 \mathrm{mM}$ DMP (dimethyl pimelimidate, Thermo Scientific) was freshly prepared and directly added to the agarose. After $1 \mathrm{~h}$ incubation, the cross-linker was quenched with $50 \mathrm{mM}$ Tris, pH 8.0. Before use, beads were washed once with $200 \mathrm{mM}$ acetic acid, $500 \mathrm{mM} \mathrm{NaCl}, \mathrm{pH}$ 2.7, and twice with $20 \mathrm{mM} \mathrm{NaP}, \mathrm{pH}$ 7.0.

HeLa cell lysate. HeLa suspension cells $\left(5 \times 10^{8}\right)$ were lysed in $8 \mathrm{ml}$ of lysis buffer (20 mM sodium phosphate ( $\mathrm{NaP}$ ), $\mathrm{pH} 7.4,150 \mathrm{mM} \mathrm{NaCl}, 1 \% \mathrm{SDS}, 1 \%$ Triton, $0.5 \%$ sodium deoxycholate, 5 mM EDTA, 5 mM EGTA, 10 mM NEM, $1 \mu \mathrm{g} / \mu \mathrm{l}$ each of aprotinin, leupeptin and pepstatin and $1 \mathrm{mM}$ Pefabloc). Alternatively (immunoprecipitation in Fig. 4b), lysis was done in PBS, 1\% SDS, 5 mM EDTA, $5 \mathrm{mM}$ EGTA, $10 \mathrm{mM}$ NEM, $1 \mu \mathrm{g} / \mu \mathrm{l}$ each of aprotinin, leupeptin and pepstatin and $1 \mathrm{mM}$ Pefabloc. Both buffers work equally well with HeLa cells. The viscous lysate was sonicated until it became fluid. The cell lysate was then supplemented with $50 \mathrm{mM}$ dithiothreitol (DTT), boiled for $10 \mathrm{~min}$ and finally diluted 1:10 with RIPA buffer without SDS (20 mM NaP, pH 7.4, $150 \mathrm{mM} \mathrm{NaCl}, 1 \%$ SDS, $1 \%$ Triton, $0.5 \%$ sodium deoxycholate, 5 mM EDTA, 5 mM EGTA, $1 \mu \mathrm{g} / \mu \mathrm{l}$ each of aprotinin, leupeptin and pepstatin, $1 \mathrm{mM}$ Pefabloc and $20 \mathrm{mM} \mathrm{NEM}$ ). The lysate was filtered through a $0.45-\mu \mathrm{m}$ filter (Rotilabo syringe filters, Carl Roth $\mathrm{GmbH}$ ) and was used for immunoprecipitation.

Mouse liver tissue lysate. Male 10-week-old C57BL/6J mice were obtained from Charles River Laboratories and maintained on a 12-h light-dark cycle with regular unrestricted diet. Animal procedures have been approved by local authorities (Regierungspräsidium Karlsruhe) and are in accordance with US National Institutes of Health guidelines. Excised livers were shock frozen and pulverized under liquid nitrogen. Tissue powder samples were lysed as above (both buffers work). The lysate was sonicated until it became fluid and all liver pieces were dissolved. After boiling and dilution, the lysate was centrifuged at $16,000 \mathrm{~g}$ for $15 \mathrm{~min}$, and the supernatant was filtered through a $0.45-\mu \mathrm{M}$ filter. Protein concentration was determined with Pierce $660 \mathrm{~nm}$ Protein Assay Reagent (Thermo scientific) containing Ionic Detergent Compatibility Reagent (Thermo Scientific) and adjusted to $1 \mathrm{mg} / \mathrm{ml}$. To each $10 \mathrm{ml}$ liver lysate, $100 \mu \mathrm{l}$ equilibrated Protein G-agarose were added and incubated for $1.5 \mathrm{~h}$ at $4{ }^{\circ} \mathrm{C}$ to deplete contaminating antibodies. Subsequently, the lysate was passed over a column to remove the agarose and was used for immunoprecipitation.

Immunoprecipitation and peptide elution. For SUMO immunoprecipitations, $100 \mu \mathrm{limmobilized} \mathrm{antibodies} \mathrm{were} \mathrm{added} \mathrm{to} \mathrm{each} 10 \mathrm{ml}$ of lysate (HeLa or mouse liver) and incubated overnight at $4{ }^{\circ} \mathrm{C}$. Beads were washed three times with $20 \mathrm{mM} \mathrm{NaP}, \mathrm{pH} 7.4,150 \mathrm{mM} \mathrm{NaCl}, 0.1 \%$ SDS, $1 \%$ Triton X-100, 0.5\% sodium deoxycholate, $5 \mathrm{mM}$ EDTA, $5 \mathrm{mM}$ EGTA, $10 \mathrm{mM}$ NEM, $1 \mu \mathrm{g} / \mu \mathrm{l}$ each of aprotinin, leupeptin and pepstatin and $1 \mathrm{mM}$ Pefabloc. A final wash with three bead volumes of high-salt buffer $(500 \mathrm{mM} \mathrm{NaCl}$ instead of $150 \mathrm{mM})$ was carried out for $30 \mathrm{~min}$ at $37^{\circ} \mathrm{C}$ on a rotating wheel. Two consecutive elution steps were performed at $37^{\circ} \mathrm{C}$ on a rotating wheel for $30 \mathrm{~min}$, each time using three bead volumes of elution buffer (high-salt buffer plus $0.5 \mathrm{mg} / \mathrm{ml}$ epitope-containing peptide (for SUMO1 21C7, VPMNSLRFLFE; for SUMO2 8A2, IRFRFDGQPI; for SUMO1 $_{76-86}$, TPKELGMEEED). Eluates were combined and precipitated with $10 \%$ TCA. The precipitated pellet was washed once with $100 \%$ acetone before it was dried and resuspended in SDS sample buffer.

Peptide competition assays. Monoclonal anti-SUMO1 and anti-SUMO2/3 antibodies were diluted in 5\% milk in PBST to a concentration of $4 \mathrm{nM}$. Candidate peptides were added at a final concentration of $8 \mu \mathrm{M}$. After $1 \mathrm{~h}$ incubation, the preincubated antibodies were used to detect $4 \mu \mathrm{g}$ recombinant SUMO1 or SUMO2 (spotted on a nitrocellulose membrane) by immunoblotting (HRPcoupled secondary antibody, ECL).

Mass spectrometry (LC-MS/MS). Eluted proteins from control immunoprecipitation, SUMO1 immunoprecipitation and SUMO2 immunoprecipitation were separated with 1D SDS-PAGE. Each lane was cut into equal pieces before undergoing in-gel digestion with trypsin as described previously ${ }^{54}$. Peptides were analyzed by liquid tandem MS (LC-MS/MS) as described previously with minor modifications $\mathbf{s}^{55}$.

Data and bioinformatics analysis. Raw MS data were analyzed by MaxQuant v1.0.13.13 (ref. 56) where MS/MS spectra were searched by Mascot search engine v2.3.2 (ref. 57) against IPI Human protein database (v3.72) supplemented with frequently observed contaminants as described previously ${ }^{58}$. Supplementary Table 1 lists proteins that were identified in both biological replicates of SUMO1 and SUMO2 immunoprecipitations. To create a high-confidence list of SUMOylated proteins, protein intensity values that are calculated from summed signal intensities of the identified peptides by MaxQuant were used as selection criteria. For each protein in the list, the percentage of intensities belonging to the respective immunoprecipitations were calculated. Proteins with less than $10 \%$ intensity in the control immunoprecipitation were used for the analysis (Fig. 5). To generate the lists 'SUMO1 0-10\% threshold' and 'SUMO2 0-10\% threshold' in Supplementary Table 1, only proteins having signal intensities that were at least $10 \%$ higher in the corresponding SUMO immunoprecipitation compared to control immunoprecipitation were kept. Proteins with background signals of $10-25 \%$ (intensities in the control immunoprecipitation) were retained in a separate list in Supplementary Table $\mathbf{1}$ and included in the analysis shown in Supplementary Figure 2.

52. Pichler, A., Gast, A., Seeler, J.S., Dejean, A. \& Melchior, F. The nucleoporin RanBP2 has SUMO1 E3 ligase activity. Cell 108, 109-120 (2002).

53. Lallemand-Breitenbach, V. et al. Arsenic degrades PML or PML-RAR $\alpha$ through a SUMOtriggered RNF4/ubiquitin-mediated pathway. Nat. Cell Biol. 10, 547-555 (2008).

54. Shevchenko, A., Tomas, H., Havlis, J., Olsen, J.V. \& Mann, M. In-gel digestion for mass spectrometric characterization of proteins and proteomes. Nat. Protoc. 1, 2856-2860 (2006).

55. Hsiao, H.H., Meulmeester, E., Frank, B.T., Melchior, F. \& Urlaub, H. "ChopNSpice," a mass spectrometric approach that allows identification of endogenous small ubiquitinlike modifier-conjugated peptides. Mol. Cell Proteomics 8, $2664-2675$ (2009).

56. Cox, J. \& Mann, M. MaxQuant enables high peptide identification rates, individualized p.p.b.-range mass accuracies and proteome-wide protein quantification. Nat. Biotechnol. 26, 1367-1372 (2008).

57. Perkins, D.N., Pappin, D.J., Creasy, D.M. \& Cottrell, J.S. Probability-based protein identification by searching sequence databases using mass spectrometry data. Electrophoresis 20, 3551-3567 (1999).

58. Nikolov, M. et al. Chromatin affinity purification and quantitative mass spectrometry defining the interactome of histone modification patterns. Mol. Cell Proteomics 10, M110.005371 (2011). 